\title{
Satellite Observations of the Equatorial Mg II Dayglow Intensity Distribution
}

\author{
J.-C. Gérard ${ }^{1}$ and A. Monfils
}

Institut d'Astrophysique, University of Liège, Ougrée-Cointe, Belgium

\begin{abstract}
Continuous measurements of the Mg II (2796-2803 $\AA$ ) doublet intensity in the equatorial dayglow have been obtained at dusk during the period March 19 to May 23, 1972, by using the TD1 A UV astronomical satellite looking upward. These data confirm that the glow observed near the equator can be accounted for by resonance scattering of sunlight by $\mathrm{Mg}^{+}$ions present at altitudes exceeding $530 \mathrm{~km}$. Isophote maps are presented showing the morphology and dynamics of the phenomena. It is clear that the emission zones are closely related to the position of the dip equator and present either a single or a double peak. Important latitudinal asymmetries are frequently observed: it is suggested that they reflect differences in the height distribution of the ion layer in the two hemispheres, in agreement with the results of ionospheric and satellite observations. A statistical analysis of longitude and planetary magnetic activity effects is made. A maximum occurrence frequency is reached in the Asian sector $\left(60^{\circ}-150^{\circ} \mathrm{W}\right)$. Important day-to-day variations are observed on some occasions, but the average daily in tensity is not affected by $K p$.
\end{abstract}

Ground-based optical observations of the equatorial red arcs near the magnetic equator date back to more than 10 years ago [Barbier and Glaume, 1960]. They have provided important information on the morphological and dynamical properties of the $\lambda 6300 \mathrm{O} I$ radiation in the vicinity of the equator (see review by Reed [1972]). Later, measurements were made at various wavelengths, thus completing the understanding of the physical and chemical processes involved. However, it is only the recent use of satellite-borne photometers and spectrometers that gives a global picture of the phenomenon.

Hicks and Chubb [1970] discovered the existence of two UV arcs symmetrically centered on the dip equator. The observed intensity was mainly accounted for by Hanson [1969] in terms of nighttime radiative recombination of $\mathrm{O}^{+}$ions at 1304-1356 $\AA$. The belt shape of these UV arcs was clearly illustrated by the photographs taken from the moon during the Apollo 16 mission [Carruthers and Page, 1972].

Observations by the Ogo 4 satellite of the night $\lambda 6300$ intensity were reported by Reed et al. [1973] and discussed by Chandra et al. [1973]. Thomas and Donahue [1972] measured with Ogo 6 the 5577- $\AA$ intensity distribution and derived the structure of the electron density. Both sets of data showed important asymmetries in the integrated intensity with respect to the dip equator. They also gave evidence of important longitudinal variations in the intensity distribution, thus suggesting some effects of the magnetic field morphology on the electron density. Both forbidden oxygen lines are produced by dissociative recombination of $\mathrm{O}_{2}{ }^{+}$, whose nighttime source depends on charge exchange of $\mathrm{O}_{2}$ with the dominant ion at the altitude of emission, $\mathrm{O}^{+}$. The arcs observed in these wavelengths are thus the optical signature of the equatorial anomaly.

The existence of $\mathrm{Fe}^{+}$ions in the equatorial $F_{2}$ region was detected by Hanson and Sanatini [1970] with the Ogo 6 retarding potential analyzer. Those authors explained the presence of these ions at such altitudes with the 'fountain effect theory,' which postulates that metallic ions from the meteorite ablation region are raised to considerable heights by $\mathbf{E} \times \mathbf{B}$

\footnotetext{
${ }^{1}$ Aspirant of the Belgian Foundation for Scientific Research.
}

Copyright (C) 1974 by the American Geophysical Union. motions. Magnesium ions have also been detected in the equatorial $\mathbf{E}$ region by rocket-borne mass spectrometers by Aikin and Goldberg [1973]. They were found in amounts comparáble to the amount of $\mathrm{Fe}^{+}$and exhibited a peak near 95 $\mathrm{km}$ reaching a density of $10^{3} \mathrm{~cm}^{-3}$. The density decreased at higher altitudes, but no results were reported above $120 \mathrm{~km}$.

Recently, Boksenberg and Gerard [1973] described the TD1 A satellite observation of the presence of an UV dayglow above $540 \mathrm{~km}$, which was mainly attributed to the resonance scattering of the Mg II 2796- to 2803-A doublet in the dusk subtropical atmosphere. The purpose of this paper is to describe the morphology of this UV dayglow on a worldwide basis and to illustrate it with isophote maps. In particular, it is shown that important interhemisphere asymmetries often exist. The influence of $K \dot{p}$ on the overall intensity is investigated and turns out to be negligible. The longitude effects are studied and are clearly present in these data.

\section{INSTRUMENTATION}

The observations have been made by means of spectrophotometers aboard an Esro satellite as part of astronomical experiment S2/S68. The satellite, labeled TD1 A, was launched on March 12, 1972, from the Western test range in California. The period was $95.5 \mathrm{~min}$, and the orbit was nearly circular (altitude of $530 \mathrm{~km}$ ) and nearly polar (inclination of $97^{\circ} 55^{\prime}$ to the earth's equator). This angle has been chosen so as to have the orbital plane precess about the rotation axis of the earth at a rate of approximately $1 \% \mathrm{~d}$, which means that the orbit is roughly sun synchronous. Figure 1 describes the orbital characteristics of TD1 for the vernal equinox configuration. The equatorial regions are crossed at approximately 0600 and 1800 LT, i.e., at dawn and dusk, respectively. This situation remains invariant, owing to the sun-synchronous character of the orbit.

The symmetry axis of the satellite lies along a plane perpendicular to the sun-satellite line. Moreover, the bottom of the spacecraft is kept facing the earth by means of horizon sensors.

The S2/S68 experiment, one of the seven experiments aboard, is composed of a relatively large telescope (27.5-cmdiameter off-axis paraboloid) followed by a spectrophotometric device. 


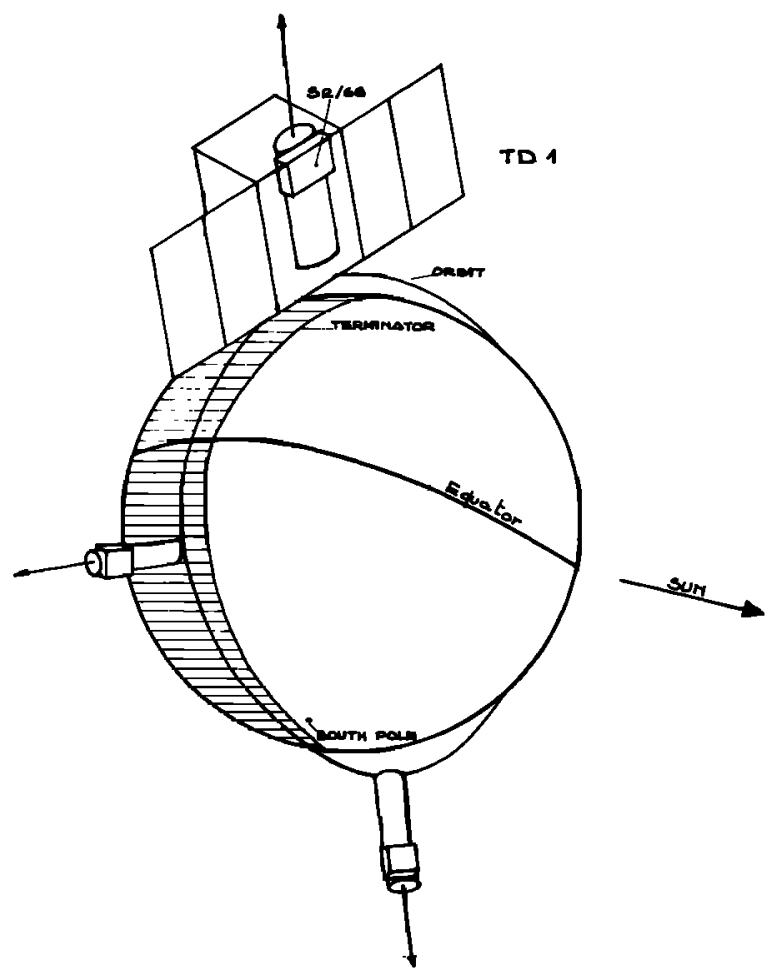

Fig. 1. Orbit of the TD1 A Esro satellite. The S2/S68 UV telescope is looking upward from about $530 \mathrm{~km}$.

A very important fact is that owing to the astronomical aims of the experiment, the telescope, whose optical axis is parallel to the symmetry axis of the satellite, is constantly pointing in a direction close to the zenith and scans great ecliptic circles. Consequently, all the data give an integration on an optical path ranging from $530 \mathrm{~km}$ to infinity. The telescope has the obvious effect of reducing the observations to a rather narrow beam. The wavelength windows are such that the region extending from $1350 \AA$ to a little less than 3000 $\ddot{A}$ is fully covered by means of four adjacent spectral channels. The wavelengths likely to give rise to some airglow (1216 and $1304 \AA$ ) have carefully been excluded.

Channel A 1 , the only one to be discussed fully below, corresponds to the passage of the beam through a filter limiting the transmission on the short-wavelength side. The RbTe photocathode photomultiplier thus limits the quantum efficiency on the long-wavelength side of the band, giving rise to a roughly Gaussian shape centered on 2750 and $300 \AA$ wide at half height. The acceptance solid angle is 33.1 arc min².

Channels A2-A4 work on a totally different principle, since the light is dispersed by a grating.

\section{BACKGROUND AND NOISE}

Our observations being part of the unexpected signals, it is important to stress the predicted and observed backgrounds. Four types of sources were foreseen: (1) scattered sunlight, (2)

TABLE 1. Noise Sources in Counts/0.148 s

\begin{tabular}{lccrr}
\hline Source & A1 & A2 & A3 & A4 \\
\hline Sunlight & 7 & & & \\
Airglow & & 25 & 20 & \\
Particles & 3 & & 3 & 3 \\
Detectors & 1 & & 1 & 1 \\
\hline
\end{tabular}

airglow and auroras, (3) high-energy particles, and (4) electronic and detector noise.

Scattered sunlight. This was a very important background noise, since it was necessary to record faint stellar spectra while the telescope was illuminated at $90^{\circ}$ by the solar light No remnant influence of scattered sunlight has ever been detected; thus any influence of the earth or the moon except for direct observations is eliminated.

Airglow. We have mentioned that the wavelengths likely to correspond to a residual airglow above $550 \mathrm{~km}$ have been excluded. This was the reason for the lower wavelength limit $(1350 \AA)$. A residual UV signal of about 20 counts per interval (apparently due to instrumentally scattered Lyman $\alpha$ ) is, however, observed in channels A2 and A3. This signal is negligible for A1 and A4.

High-energy particles. The detectors have been protected from trapped particles by shielding of the tube walls. The result is roughly as was expected: in the South Atlantic anomaly (SAA) an important background develops, and for channels $\mathrm{A} 1, \mathrm{~A} 3$, and $\mathrm{A} 4$ it prevents any sensitive detection of light. For channel A2 the apparent fluorescence caused in the window is not detected by the CsI photocathode. This channel remains almost insensitive to the particles.

Outside the SAA, except in the auroral regions, the particle noise in all channels is negligible. This noise is easy to separate from the preceding noises by closing the entrance shutter; thus any light is prevented from reaching the photomultipliers.

Detector noise. Laboratory tests have shown that the preamplifier noise is negligible in comparison with P.M. dark currents. The P.M. dark currents are in turn negligible with respect to the airglow and particle noises, which along with some scattered solar light in Al, appear to be the only effective ones.

Table I gives for the equatorial regions outside the anomaly the distribution of noise counts among the various sources cited above. Figure 2 illustrates the sensitivity threshold as a function of wavelength, as determined by the total noise.

\section{ObSERVATIONS}

The observations analyzed in the present paper cover 75 days of the first summer scanning of the satellite. This period

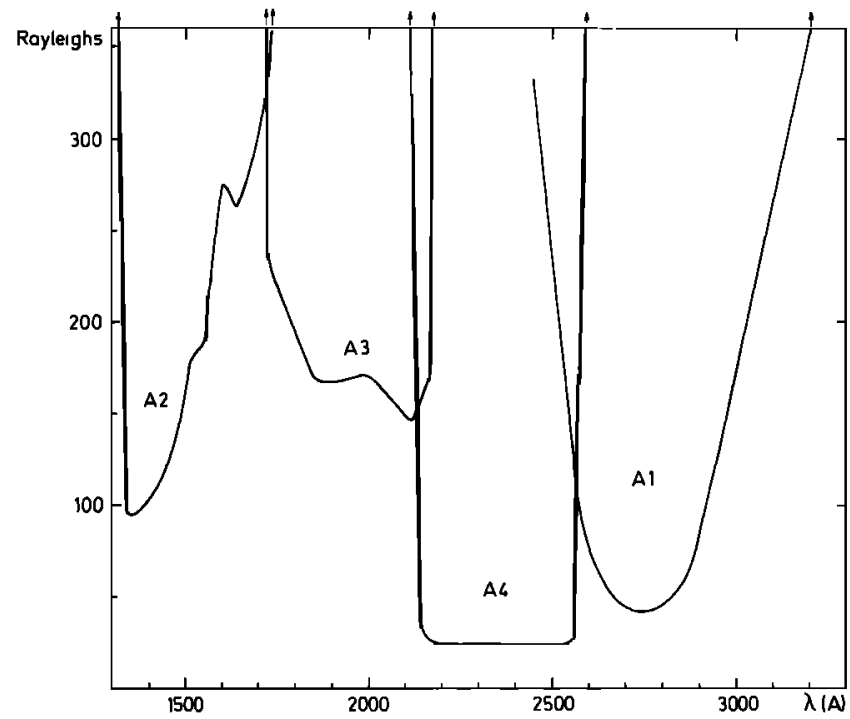

Fig. 2. Sensitivity threshold of the four channels in rayleighs plotted versus wavelength. 


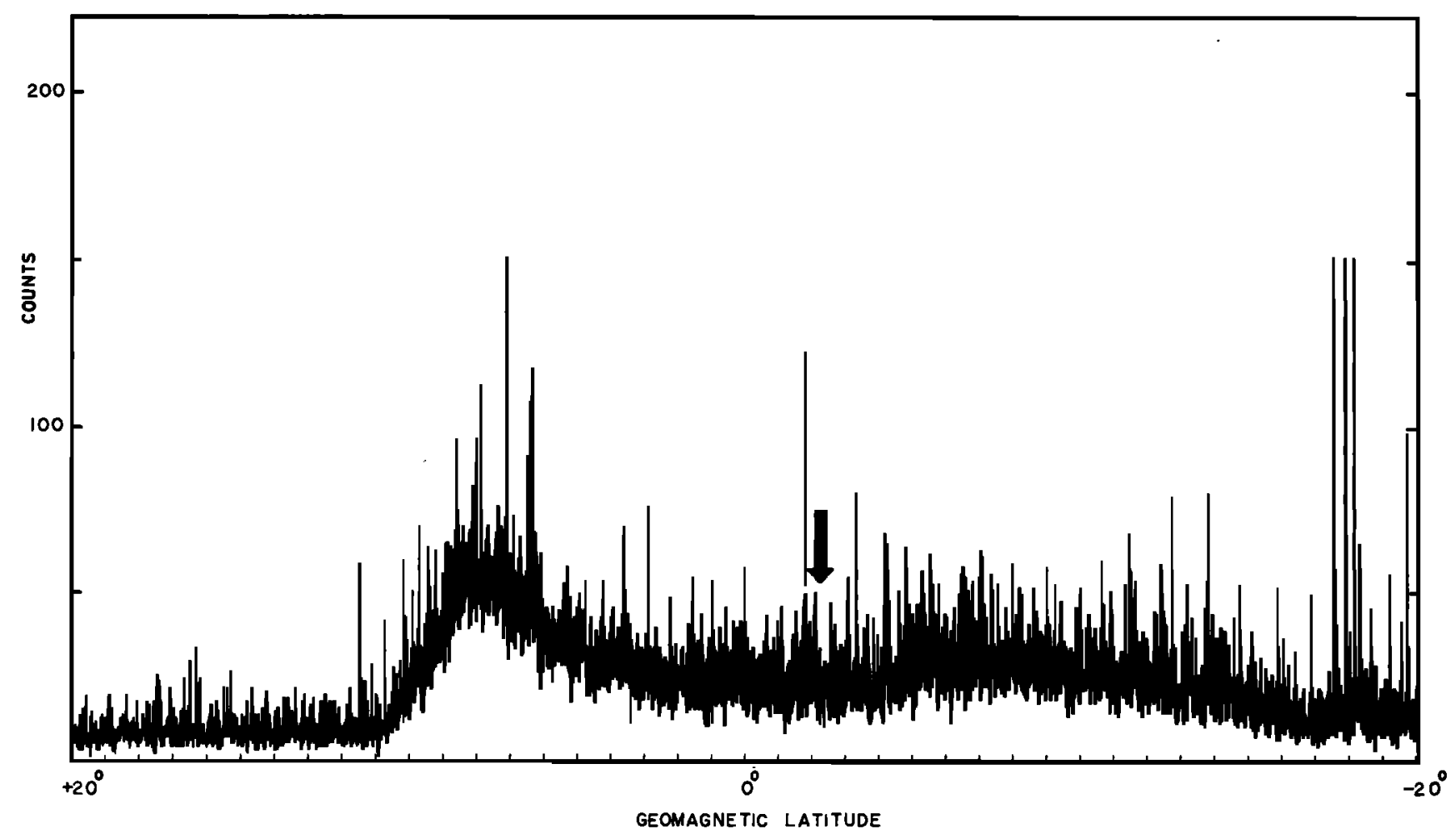

Fig. 3. Example of the signal observed during a dusk equator crossing on April 12, 1972, at 1734 LT during orbit 473. Here the altitude is $530 \mathrm{~km}$, and the longitude is $111^{\circ} \mathrm{E}$. The broad arrow indicates the position of the magnetic dip equator.

has been chosen because it corresponds to the correct functioning of the tape recorder of the satellite, which in turn guarantees complete orbit recordings and a large amount of successive equatorial scans.

The data, an example of which is reproduced in Figure 3, appear as increases of the continuous background in channel A l, i.e., for the bandwidth of $2750 \pm 150 \AA$ during the dusk crossing of the equatorial region. It is very important to stress the general absence of any signal on the three spectrophotometric channels.

Although we shall not be analyzing here the results of our fellow experiment S59, it must be mentioned that equatorial signals have also been recorded by S59 in all three of their channels. Consequently, the possible wavelength range of the emitted light has been discussed in an earlier publication [Boksenberg and Gérard, 1973] and has been shown to extend from 2580 to $2800 \AA$. By considering the various possibilities of spectral identification of the emitting species the glow has finally been attributed to the strong resonance transition of $\mathrm{Mg}$ II lying at $\lambda 2796-2803 \AA$. Resonance scattering of sunlight by $\mathrm{Mg}^{+}$ions can account for the majority (or totality) of the observed signal. This presence at the end of the day and the absence of any detectable signal during the dawn crossing of the equator are in agreement with the fountain effect theory developed by Hanson et al. [1972].

If the signal observed is entirely ascribed to the $\mathrm{Mg}$ II doublet, the detection sensitivity is $5.6 \mathrm{R}_{\text {count }}{ }^{-1}$, or $4.410^{7}$ $\mathrm{Mg}^{+}$ion $\mathrm{cm}^{-2}$ count $^{-1}$, as established in the previous paper.

\section{MAPS}

Isophote maps have been found to illustrate best the localization, structure, and dynamics of this UV dayglow. The data collected during 1 day of observation are displayed on planispheres in geodetic coordinates.
Owing to the rotation of the earth under the orbital plane the whole sphere is covered by 15 or occasionally 16 successive orbital traces. The intensity of the airglow observed in the region extending $\pm 30^{\circ}$ from the geographic equator is examined except for the SAA region, where the very high particle noise prevents the detection of the dayglow signal. The boundaries of this region are indicated on each map.

Since each equator crossing is spaced by nearly $24^{\circ}$ in longitude, thus causing an apparent motion westward, the accuracy of the morphology is of the same order of magnitude. On each map are indicated the date, orbit numbers, and the sum over 24 hours of the 3-hour planetary indices, $\sum K p$.

As was mentioned above, the local time of all observations is nearly constant and remains close to $1800 \mathrm{LT}$. Owing to the 22.5-hour delay between the first and the last satellite passes used in each display, reproducibility of the forms at a given longitude is not to be expected on successive maps. Furthermore, the equatorial passes are spaced by about $2^{\circ}$ in longitude from those of the previous day.

The magnetic dip equator at $600 \mathrm{~km}$ is plotted after Cain's [1969] Pogo model adjusted to 1972. All orbits used to draw these maps are southbound, since we consider only the dusk equator crossings. Each map starts with the first pass situated westward of the SAA region. Three sets of two consecutive maps are illustrated by Figures 4-6. On two of the figures, one satellite pass is missing so far, thus giving rise to a gap nearly $44^{\circ}$ wide in longitude. As can readily be seen, the forms are generally aligned about the magnetic dip equator, thus suggesting that the species causing the airglow is ionized. This alignment is particularly remarkable in the Pacific sector $\left(160^{\circ} \mathrm{E}-80^{\circ} \mathrm{W}\right)$, where the magnetic declination is eastward. An exception to this rule can be found in Figure $6 b$, where the form is aligned parallel to the geographic equator.

The total latitudinal extension (as determined by the sen- 


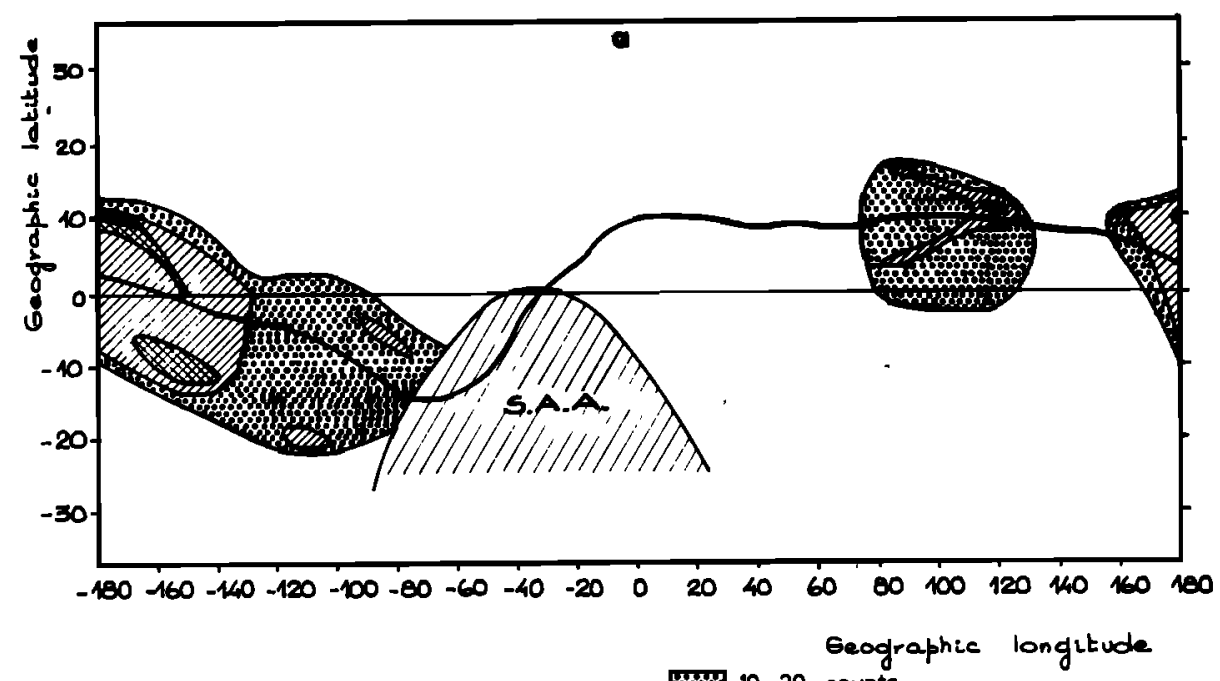

Orbits $300-314$

wo 10 counts

March 31 - Apr 1, 1972

Z20-40 counts

IKp . 23 *

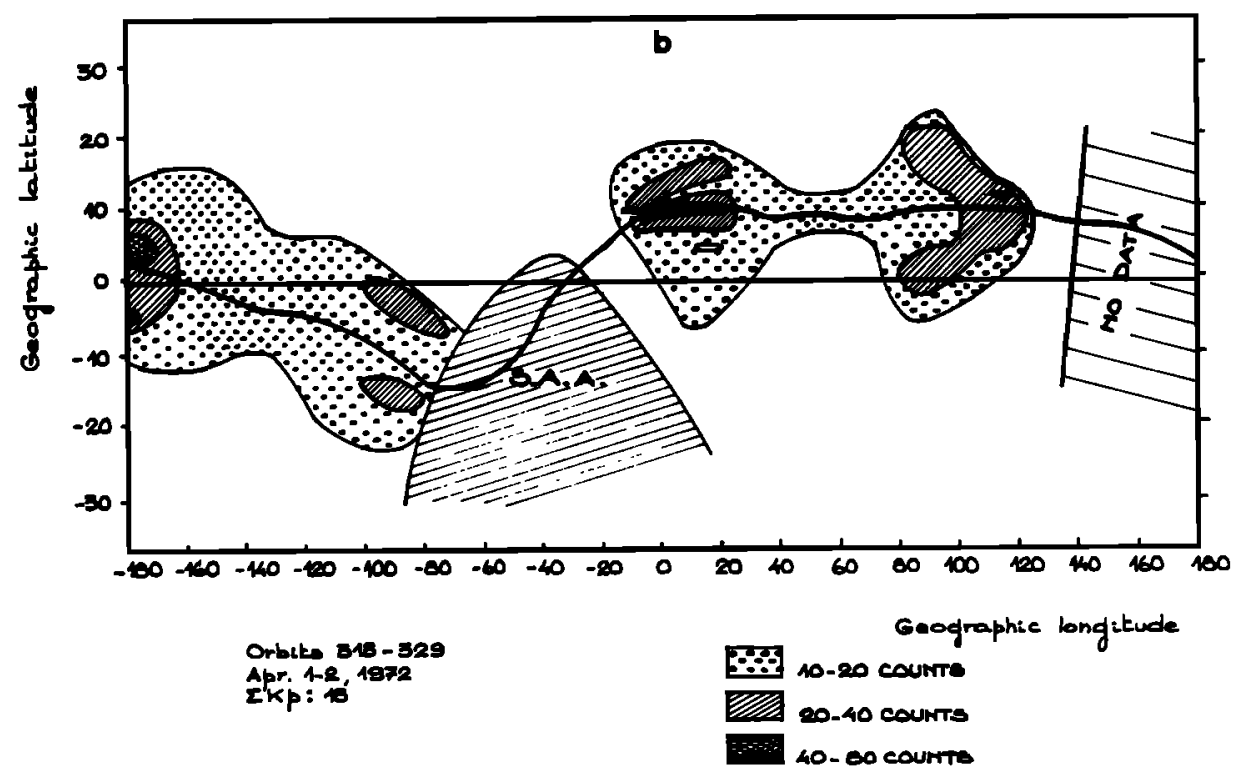

Fig. 4. Isophote maps of the UV Mg II dayglow plotted in geodetic coordinates for (a) orbits 300-314 and (b) orbits 315-329. The shaded area is the South Atlantic anomaly (SAA) region, where the particle noise prevents airglow measurements.

sitivity threshold of the instrument) never exceeds $30^{\circ}$ but varies strongly with time and longitude. Most striking are the gaps observed in some sectors, principally in the American one. The degree of conjugacy between hemispheres is occasionally high (Figure $4 b$ ) but is generally poor. Figure $5 b$ illustrates the case of an arc in the Pacific southern hemisphere that has no conjugate form in the other hemisphere. A minimum of intensity corresponding to the ionospheric equatorial trough is sometimes observed on the dip equator (Figure 5a).

Furthermore, the southern arc is generally stronger than the northern one during April and May in the Pacific region. This lack of conjugacy is ascribed to differences of altitude of the ion distribution north and south of the dip equator, causing the column ion density above the satellite to be different in the two hemispheres. Such a latitudinal dissymmetry has been observed in the height of the $F_{2}$ peak by ionospheric sound- ings [Lyon and Thomas, 1963] and has been invoked by Chandra et al. [1973] in the case of equatorial red arcs. The altitude effect is possibly caused by transequatorial winds blowing from the summer hemisphere.

Figures $4(a, b)$ and $5(a, b)$ show that the morphology is occasionally persistent from day to day, at least in the Pacific sector. On the contrary, Figure $6(a, b)$ illustrates an example of dramatic variation, although the magnetic activity remains moderate during both days.

\section{LONGITUDINAL VARIATIONS}

Longitudinal effects are readily apparent in the discontinuities observed on the maps shown in Figures 4-6. However, the location of the region with no signal varies from day to day. Consequently, all data so far reduced have been added in sectors $30^{\circ}$ wide in longitude, and the relative frequencies of occurrence have been calculated in each sector in 

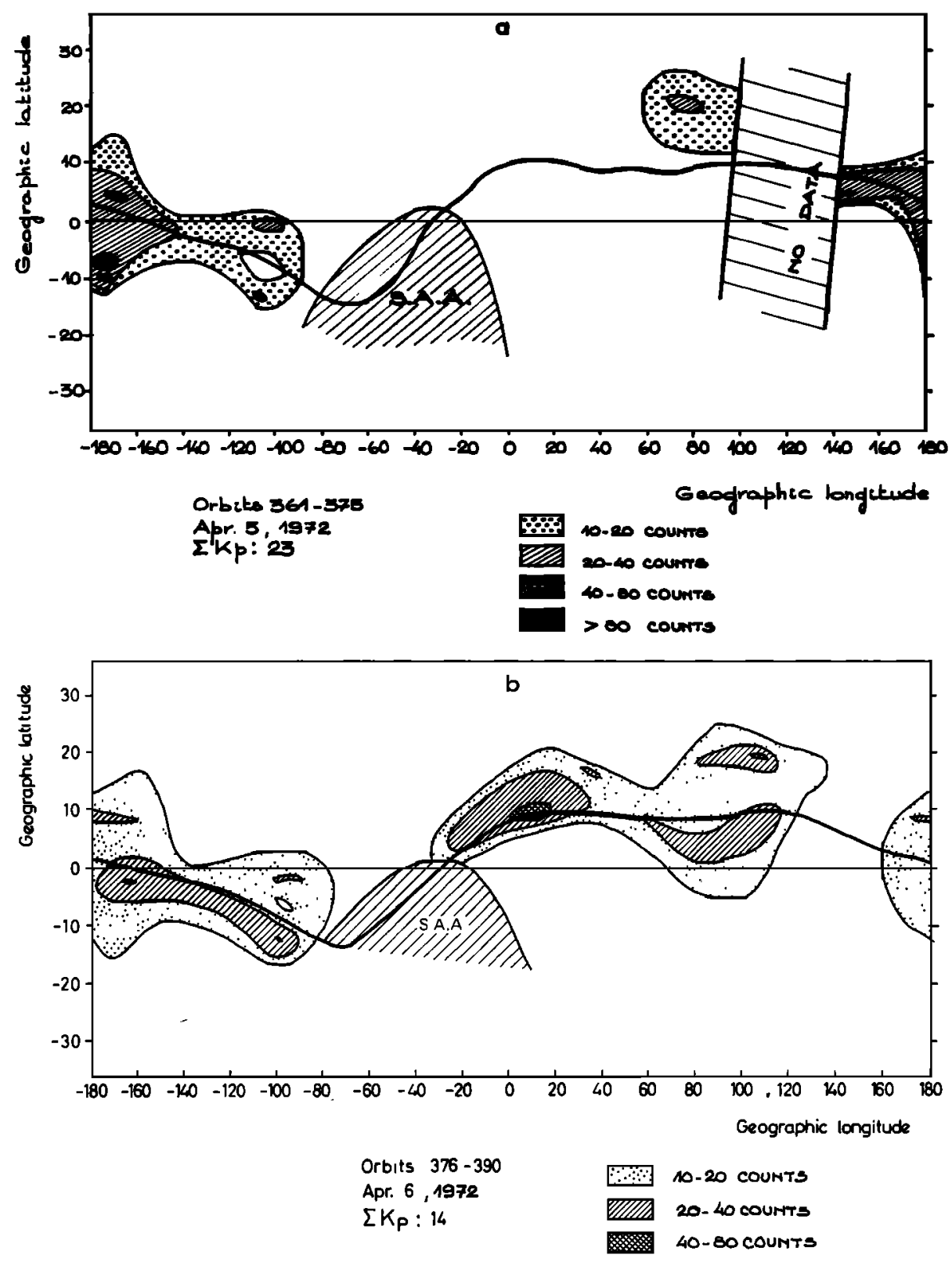

Fig. 5. Isophote maps of the UV Mg II dayglow plotted in geodetic coordinates for $(a)$ orbits $361-375$ and $(b)$ orbits $376-390$. The shaded area is the SAA region, where the particle noise prevents airglow measurements.

order to determine whether statistical longitudinal effects would appear. The histogram illustrating this result is plotted in Figure 7. It is based on more than 600 good-quality equatorial passes. A deep minimum is observed in the American zone $\left(0^{\circ}-90^{\circ} \mathrm{W}\right)$. Since the range of latitude covered in the $30^{\circ}-90^{\circ} \mathrm{W}$ sector is less than that in the other sectors, owing to perturbations by particles of the SAA, the statistical results are unreliable in this region. However, we believe the dip to be real, since it is also present in the adjacent unperturbed sector. A large maximum is apparent in the Asian sector $\left(60^{\circ}-120^{\circ} \mathrm{E}\right)$ in $85 \%$ of the favorable observations.

These longitudinal variations are most likely connected to the declination angle, the mutual position of the dip and the geographic equator, and the strength of the magnetic field $\mathbf{B}$. For a given value of the $\mathbf{E}$ field the drift velocity $W=$ $\mathbf{E} \times \mathbf{B} / B^{2}$ is maximum in the American zone, where $B$ is minimum, but it has been shown above that the observed effect works in the opposite way.

Observations relevant to this problem have been described by Lyon and Thomas [1963], who have shown from ionospheric data that the equatorial anomaly develops earlier in the Asian sector than in the American sector. This has recently been confirmed by the Ariel 3 satellite electron density measurements [Hopkins, 1972], which have demonstrated the anomaly to begin between 500 and $600 \mathrm{~km}$ nearly 3 hours earlier in the Asian sector than in the American one. Accordingly, it is likely that differences in the diurnal variations of the $\mathbf{E}$ field exist at various longitudes, thus causing differences in the altitude reached at a given local time by the ions. The measurements reported here integrate upward from about 530 $\mathrm{km}$; they are consequently very sensitive to such altitude variations, which are observed as longitudinal effects. The problem of why the anomaly starts developing at local times 

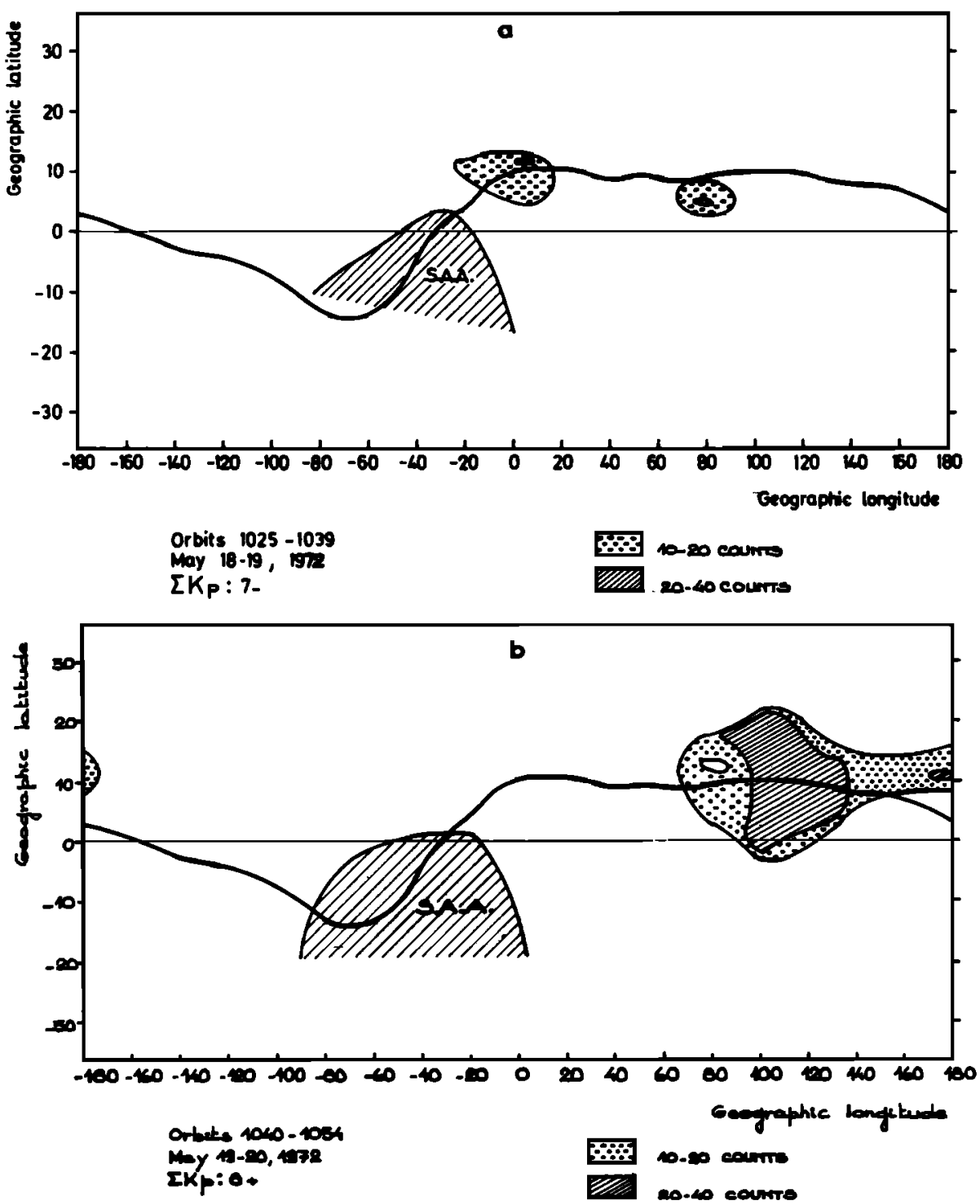

Fig. 6. Isophote maps of the UV Mg II dayglow plotted in geodetic coordinates for $(a)$ orbits 1025-1039 and $(b)$ orbits 1040-1054. The shaded area is the SAA region, where the particle noise prevents airglow measurements.

dependent on longitude is outside the scope of this work: it is likely to be related to the dynamo theory itself. It should be stressed that all the results described in this section concern quiet magnetic days $(K p<3)$.

\section{Kp EFFECTS}

The influence of the magnetic activity planetary index $K p$ daily variation on the overall intensity has been investigated. The frequency of observation of the UV dayglow at all longitudes has been compared for days on which $K p$ was less than or greater than 3, the usual limit between quiet and disturbed days.

The observed frequencies are $43 \%$ when $K p<3$ and $44 \%$ when $K p>3$, thus demonstrating incidentally that the presence of metallic ions at such considerable heights is not unusual. Accordingly, it is concluded that the planetary magnetic activity has no noticeable effect on the total intensity observed.

\section{SUMMARY}

All the characteristics of the UV dayglow discussed here are consistent with its being ascribed to resonance scattering of
$\mathrm{Mg}^{+}$ions in sunlight. Isophote maps were found to be convenient to illustrate the morphology and temporal variations of the glow. It turns out that the arcs can be either single or double but are generally aligned parallel to the magnetic dip equator. Important interhemisphere dissymmetries are often observed, although conjugacy can occasionally be found in some sectors.

Seasonal variations are not clearly demonstrated by these measurements; however, the winter hemisphere arc is generally stronger in the Pacific sector. Gaps along the equator are very frequently observed. When these longitudinal variations are analyzed statistically, they show a sinuslike distribution having a maximum occurrence frequency in the Asian sector $\left(60^{\circ}-120^{\circ} \mathrm{E}\right)$ and a dip in the American sector $\left(0^{\circ}-90^{\circ} \mathrm{W}\right)$. This finding is consistent with the electron density measurements, which show the equatorial anomaly to develop earlier in the Asian zone. The dissymmetries and longitudinal variations are possibly due to differences in the altitude of the ion layer. Vertical metallic ion distribution profiles as well as more quantitative information regarding winds and the influence of the magnetic field morphology on the dynamo $\mathbf{E}$ field are needed to clarify this problem. 


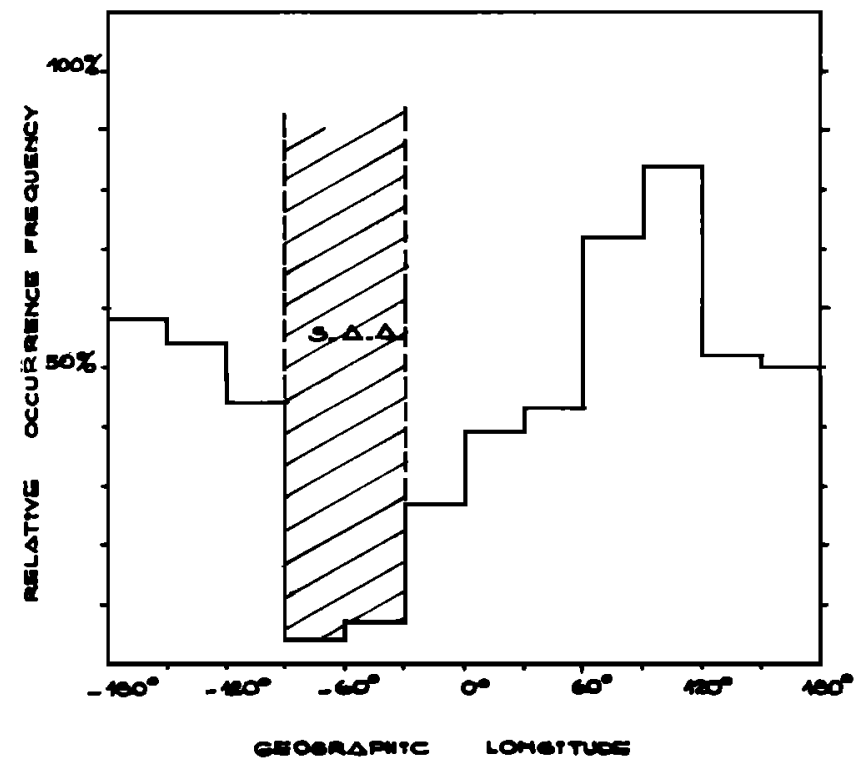

Fig. 7. Longitudinal variation of the relative occurrence frequency of the equatorial glow for magnetically quiet days.

Acknowledgments. C. Thiry is gratefully acknowledged for her help in data reduction.

The Editor thanks W. B. Hanson and P. Mange for their assistance in evaluating this report.

\section{REFERENCES}

Aikin, A. C., and R. A. Goldberg, Metallic ions in the equatorial ionosphere, J. Geophys. Res., 78, 734. 1973.
Barbier, D., and J. Glaume, Les radiations de l'oxygène 6300 et 5577 $\AA$ de la luminescence du ciel nocturne dans une station de basse latitude, Ann. Geophys., 16, 319, 1960.

Boksenberg, A., and J.-C. Gérard, Ultraviolet observations of equatorial dayglow above the $F_{2}$ peak, $J$. Geophys. Res., 78, 4641, 1973.

Cain, J. C., The location of the dip equator at $E$ layer altitude, Radio Sci., 4, 781, 1969.

Carruthers, G. R., and T. Page, Apollo 16 far-ultraviolet camera/spectrograph earth observations, Science, 177, 788, 1972.

Chandra, S., E. I. Reed, B. E. Troy, and J. E. Blamont, The equatorial airglow and the ionospheric geomagnetic anomaly, J. Geophys. Res., 78, 4630, 1973.

Hanson, W. B., Radiative recombination of atomic ions in the nighttime region, J. Geophys. Res., 74, 3720, 1969.

Hanson, W. B., and S. Sanatini, Meteoritic ions above the $F_{2}$ peak, $J$. Geophys. Res., 75, 5503, 1970.

Hanson, W. B., D. L. Sterling, and R. F. Woodman, Source and identification of heavy ions in the equatorial $F$ layer, J. Geophys. Res., 77, 5530, 1972.

Hicks, G. T., and T. A. Chubb, Equatorial aurora/airglow in the far ultraviolet, J. Geophys. Res., 75, 6233, 1970.

Hopkins, H. D., Longitudinal variation of the equatorial anomaly, Planet. Space Sci., 20, 2093, 1972.

Lyon, A. J., and L. Thomas, The $F_{2}$-region equatorial anomaly in the African, American and east Asian sectors during sunspot maximum, J. Atmos. Terr. Phys., 25, 373, 1963.

Reed, E. I., Bibliography of observations of OI $6300 \AA$ night airglow at latitudes from $40^{\circ} \mathrm{N}$ to $60^{\circ} \mathrm{S}$, GSFC $X-625-72-172$, Goddard Space Flight Center, Greenbelt, Md., 1972.

Reed, E. I., W. B. Fowler, and J. E. Blamont, An atlas of low-latitude 6300-A O I night airglow from Ogo 4 observations, $J$. Geophys. Res., 78, 4630, 1973.

Thomas, R. J., and T. M. Donahue, Analysis of Ogo 6 observations of the O I 5577- $\AA$ tropical nightglow, J. Geophys. Res., 77, 3557, 1972.

(Received October 5, 1973;

accepted February 11, 1974.) 